\title{
ESTUDIO BRIOLÓGICO DE LA SIERRA DEL TORCAL DE ANTEQUERA (MÁLAGA)
}

\author{
J. Varo, ${ }^{*}$ J. Guerra** \& J. A. Gil*
}

\section{RESUMEN}

Se citan para el Torcal de Antequera setenta y siete especies de briófitos, de las cuales sesenta y ocho pertenecen a la clase Musci y nueve a la clase Hepaticae. Se citan por primera vez en Andalucía oriental: Radula lindbergiana Got. y Cololejeunea rossettiana (Mass.) Schiff, siendo la segunda localidad de Andalucía donde se cita Neckeradelphus menziesii (Hook.) Steere. Se realiza igualmente un estudio sociológico de las principales comunidades briofíticas de la zona, proponiendo como nuevas las asociaciones Neckeradelphetum menziesii y CololejeuneoRhynchostegietum tenellae.

\section{SUMMARY}

Seventy seven species of bryophytes, syxty eight belonging to the Musci class and the remaining nine to the Hepaticae, all of them collected for the first time in the area of El Torcal, are mentioned in this report. The species, Radula lindbergiana Got. and Cololejeunea rossetiana (Mass.) Schiff, are found in eastern Andalusia for the first time, being the area of El Torcal de Antequera the second site in Andalusia where the Neckeradelphus menziesii (Hook.) Steere is located. Also, a sociologic study of the most important bryophyte communities round this area is made, proposing as new the associations: Neckeradelphetum menziesii and Cololejeuneo-Rhynchostegietum tenellae.

\section{INTRODUCCIÓN}

La sierra del Torcal de Antequera se encuentra situada en el conjunto de las sierras béticas entre las de la Chimenea al O. y la de las Cabras al E. La sierra del Torcal se apoya en la sierra Pelada, situada al N.NE. a cuyo pie nace el río de la Villa, dicha sierra también ha sido estudiada por nosotros, pues entre ambas difícilmente puede establecerse una discontinuidad ni geológica ni geográfica. Por el S. la sierra del Torcal aparece como un alto paredón cortado a pico y coronada por una alta cresta, las Ventanillas, entre 1.200 y $1.300 \mathrm{~m}$ de altitud.

$\left(^{*}\right)$ Departamento de Botánica. Facultad de Ciencias. Universidad de Granada.

(**) Departamento de Botánica. Facultad de Ciencias. Universidad de Málaga. 
Este gran bloque calizo del Jurásico se presenta en estratos delgados, alternando una caliza fina cristalina o blanca oolítica y pisolítica con otra margosa brechoide, que forma lentejones o fajas horizontales y que contribuye a dar un aspecto característico al paisaje.

Una nota dominante es su extraordinaria aridez, debido a la densa fisuración de la roca y la multitud de embudos, sumideros y tubos de disolución las aguas de lluvia se filtran por una apretada red de conductos que atraviesan el espesor de la formación caliza.

El primer esłabón en la génesis de los suelos lo forman las rocas calizas, constituidas principalmente por carbonato cálcico, carbonato cálcicomagnésico y un residuo de silicatos. Los agentes erosivos, agua, viento, insolación, etc. actúan en la realización del modelado cárstico aprovechando fisuras, diaclasas, etc. disolviendo el carbonato cálcico y dejando como residuo a los silicatos. En estrecha relación con estas acciones actúan los agentes biológicos como musgos y liquenes. Bajo los primeros se reconoce el desarrollo de un suelo inicial y aue sería una protorendsina. En un grado de evolución más avanzado, se encuentran otros suelos que serían mull rendsinas. En las grietas de paredes elevadas, callejones profundos, etc. este suelo evoluciona hacia un lehm rojo de roca caliza o terra rossa. Cuando la fase emigrante de estos suelos va a parar a una torca, queda al descubierto y expuesta a la acción solar, evolucionando hacia lo que se considera una rendsina pardeada. En todos los casos el $\mathrm{pH}$ de estos suelos oscila entre 7,9 y 6,5 .

La carencia de estaciones climatológicas en la zona estudiada ha motivado a J. Delgado en su trabajo Estudio Hidrogeológico del Torcal de Antequera, a tomar los datos correspondientes a pluviosidad en las estaciones de Antequera, la Yedra, Casilla del Hierro, Villanueva de la Concepción, Los Boliches y Bobadilla Estación, extrapolando los datos posteriormente al macizo montañoso, obteniendo por el método de las isoyetas una pluviosidad que oscila entre los 700 en las cotas más bajas a 1.200 en las más altas. Estos valores de pluviometría, pueden parecer en principio un poco altos, sin embargo, los frecuentes vientos que soplan casi siempre del S. y SO. que no encuentran una barrera montañosa elevada antes de llegar al Torcal y la relativa cercanía del mar, da como resultado que sea en este macizo montañoso donde se descargue mayor cantidad de agua.

Las mismas condiciones dan lugar en el Torcal a nieblas frecuentes y muy densas permaneciendo la cumbre envuelta en ellas durante numerosos días, condiciones estas que son acusadas por la vegetación muscinal por encima de los $1.000 \mathrm{~m}$. No existen datos sobre los días de nieblas anuales. 
La única estación de las utilizadas que posee datos termométricos es la de Bobadilla Estación que se encuentra alejada del macizo y a unos 380 m s.n.m., por lo tanto la realización de un diagrama ombrotérmico no tendría un valor significativo para nuestra zona, pues implicaría la aparición de errores debidos a la topografía elevada de la sierra del Torcal.

Ante la carencia de datos y atendiendo a la vegetación de la zona se podría considerar el clima del Torcal de Antequera como un mediterráneo semi-húmedo, donde a relativas altas precipitaciones invernales, suceden meses de estiaje pronunciado, manteniendo las nieblas una alta humedad relativa, durante los días de nula precipitación.

\section{CATÁLOGO FLORÍSTICO}

\section{MUSCI}

\section{FISSIDENTALES}

\section{FISSIDENTACEAE}

Fissidens cristatus Wils. \& Mitt.

Hendiduras de rocas rezumantes. A $1.300 \mathrm{~m}$.

Mesófila, esciófila, terri-saxícola, indiferente.

Común en toda la Península Ibérica.

Circumboreal.

Fissidens minutulus Suel.

Rocas rezumantes. A $1.300 \mathrm{~m}$.

Mesófila, esciófila, saxícola, indiferente.

Toda la Península Ibérica.

Euratlántica.

\section{DICRANALES}

\section{DITRICHACEAE}

Cheilothela chloropus (Brid.) Lindb.

Torcal de Antequera (ALLORGE, V. et P., 1946).

Fisura de roca con suelo. A $1.100 \mathrm{~m}$.

Xerófila, fotófila, terri-saxícola, indiferente.

Centro y Sur de la Península Ibérica.

Submediterránea-subatlántica. 
Encalypta vulgaris Hedw.

var mutica Brid.

En suelos calizos.

Xerófila, fotófila, terri-saxícola, basófila.

Toda la Península Ibérica.

Circumboreal mesoterma.

\section{POTTIACEAE}

Aloina aloides (Schultz) Kindb.

Xerófila, fotófila, terrícola, basófila.

Toda la Península Ibérica.

Circumboreal.

Barbula acuta (Brid.) Brid.

Sobre suelos calizos. A $1.100 \mathrm{~m}$.

Xerófila, fotófila, terrícola, basófila.

Común en toda la Península Ibérica.

Circumboreal mesoterma.

Barbula fallax Hedw.

Fisuras de rocas con suelo. (ALLORGE, V. et P., 1946).

Xerófila, fotófila, terrícola, basófila.

Común en toda la Península Ibérica.

Circumboreal mesoterma.

Barbula hornschuchiana Sch.

Fisuras de rocas con suelo. Común.

Xerófila, fotófila, terri-saxícola, basófila.

Toda la Península Ibérica.

Submediterránea-subatlántica. 
Barbula unguiculata Hedw.

En fisuras de rocas con suelo.

Xerófila, fotófila, terrícola, basófila.

Toda la Península Ibérica.

Circumboreal-mesoterma.

Barbula vinealis Brid.

En fisura de roca.

Xerófila, fotófila, terri-saxícola, basófila.

Frecuente en toda la Península Ibérica.

Submediterránea-subatlántica.

Cinclidotus aquaticus (Jaeq.) B. e.

Nacimiento de la Villa, en aguas de fuerte corriente. A $800 \mathrm{~m}$.

Higrófila, fotófila, saxícola, calcícola.

Toda la Península Ibérica.

Circumboreal disyunta.

Crossidium squamigerum (Viv.) Jur.

En rocas expuestas. Frecuente.

Xerófila, fotófila, saxícola, basófila.

Toda la Península Ibérica.

Circumboreal.

Dialytrichia mucronata (Brid.) Limpr.

Sobre roca expuesta.

Higrófila, fotófila, saxícola, calcícola, indiferente.

Distintas localidades de la Península Ibérica.

Atlántico-mediterránea.

Didymodon rigidulus Hedw.

Barbula rigidula (Hedw.) Milt.

Fisuras de rocas con suelo.

Mesófila, esciófila, terri-saxícola, basófila.

Distintos puntos de la Península Ibérica.

Circumboreal-mesoterma. 
Didymodon trifarius (Hedw.) Roehl.

Didymodon luridus Hornsch.

Fisuras de rocas con suelo.

Mesófila, fotófila, saxícola, basófi'a.

Toda la Península Ibérica.

Eucladium verticillatum (Smith.) B. e.

Rocas rezumantes poco iluminadas. A $1.200 \mathrm{~m}$.

Higrófila, foto-esciófila, saxícola, basófila.

Toda la Península Ibérica.

Circumboreal, submediterránea en Europa.

Gymnostomum calcareum Nees. et Hornsch.

Torcal de Antequera (ALLORGE, V. et P., 1946).

Rocas calcáreas umbrías.

Higro-mesófila, esciófila, saxícola, basófila.

Toda la Península Ibérica.

Cosmopolita.

Pleurochaete squarrosa (Brid.) Lindb.

Torcal de Antequera (ALLORGE, V. et P., 1946).

En céspedes calizos.

Xerófila, fotófila, terri-arenícola, calcícola.

Este y Sur de la Península Ibérica.

Circumboreal-submediterránea.

Tortella inclinata (Hedw.) Limpr.

Torcal de Antequera (ALLORGE, V. et P., 1946).

Fisuras de rocas calizas.

Xerófila, fotófila, terri-arenícola, calcífila.

Común en toda la Península Ibérica.

Eurasiática-submediterránea.

Tortella nitida (Lindb.) Broth.

Torcal de Antequera (ALLORGE, V. et P., 1946).

En rocas calizas.

Xerófila, fotófila, saxícola, basófila.

Común en toda la Península Ibérica.

Circumboreal. 
Tortella tortuosa (Hedw.) Limpr.

Torcal de Antequera (ALLORGE, V. et P., 1946).

Fisuras de rocas calizas.

Xerófila, fotófila, esciófila, terri-saxícola, basófila.

Toda la Península Ibérica.

Circumboreal mesoterma.

Tortella tortuosa (Hedw.) Limpr.

fo. fragilifolia Moenk.

T. tortuosa (Hedw.) Limpr.

var. fragilifolia (Jur.) Limpr.

Sobre roca caliza con suelo.

Xerófila, foto-esciófila, terri-saxícola, basófila.

Toda la Península Ibérica.

Circumboreal-mesoterma.

Tortula inermis (Brid.) Mont.

Fisuras de rocas con suelo.

Xerófila, foto-esciófila, terri-saxícola, basófila.

Toda la Península Ibérica.

Europea.

Tortula intermedia (Brid.) De Not.

T. montana (Nees) Lindb.

Torcal de Antequera (ALLORGE, V. et P., 1946).

Fisuras de rocas calizas.

Xerófila, fotófila, terri-saxícola, basófila.

Común en toda la Península Ibérica.

Circumboreal.

Tortula muralis Hedw.

Rocas expuestas, sobre los $1.200 \mathrm{~m}$.

Xerófila, fotófila, saxícola, indiferente.

Toda la Península Ibérica.

Cosmopolita.

Tortula ruralis (Hedw.) Gaertn.

Torcal de Antequera (ALLORGE, V. et P., 1946).

Fisuras de rocas con suelo.

Xerófila, fotófila, terri-saxícola, arenícola, indiferente.

Toda la Península Ibérica.

Cosmopolita. 
Tortula ruralis (Hedw.) Gaertn.

var ruraliformis (Besch.) Wild.

T. ruraliformis (Besch.) Ingh.

Torcal de Antequera (ALLORGE, V. et P., 1946).

Fisuras de rocas con suelo.

Xerófila, fotófila, arenícola, indiferente o basófila.

Zonas litorales y algunos lugares xéricos del interior.

Circumboreal.

Tortula subulata Hedw.

Fisuras de rocas con suelo.

Xerófila, mesófila, foto-esciófila, terri-saxícola, basófila.

Toda la Península Ibérica.

Circumboreal-mesoterma.

Trichostomun crispulum Bruch.

Rocas expuestas.

Xerófila, fotófila, terri-saxícola, basófila.

Toda la Península Ibérica.

Submediterránea.

Weisia controversa Hedw.

Fisuras de rocas con suelo. A $1.200 \mathrm{~m}$.

Xerófila, fotófila, terri-saxícola, basófila.

Toda la Península Ibérica.

Cosmopolita.

GRIMMIALES

GRIMMIACEAE

Grimmia pulvinata (Hedw.) Sm.

Torcal de Antequera en rocas descubiertas (ALLORGE, V. et P., 1946).

Muy frecuente en toda la zona.

Xerófila, fotófila, saxícola, indiferente.

Toda la Península Ibérica.

Cosmopolita. 


\section{Grimmia trichophylla Grev.}

Fisuras de rocas expuestas.

Mesófila, esciófila, saxícola, calcífuga.

Diversos puntos de la Península Ibérica.

Circumboreal.

Schistidium apocarpum (Hedw.) B. e.

subsp. atrofuscum (Schimp.) Loesk.

Grimmia apocarpa Hedw.

subsp. atrofusca Schpr.

Rocas expuestas.

Xerófila, fotófila, saxícola, indiferente o basófila.

Común en toda la Penínstila Ibérica.

Cosmopolita.

\section{FUNARIALES}

\section{FUNARIACEAE}

Funaria calcarea Wahlenb.

var. mediterranea (Lindb.) C. Jens et Medel.

F. mediterránea Lindb.

Fisuras de rocas con suelo.

Xerófila, fotófila, terri-humícola, basófila.

Toda la Península Ibérica.

Submediterránea-subatlántica.

\section{ORTHOTRICHALES}

ORTHOTRICHACEAE

Orthotrichum anomalum Hedw.

var. saxatile Mild.

Torcal de Antequera (ALlORGE, V. et P., 1946).

Xerófila, fotófila, saxícola, indiferente.

Muy común.

Circumboreal. 
Orthotrichum cupulatum Hoffn.

Rocas calizas. Frecuente.

Xerófila, fotófila, saxíco'a, basófila.

Común en la Península Ibérica.

Circumpolar y atlántica.

Orthotrichum cupulatum Brid.

var. sardagnanum (Vent.) Vent.

O. sardagnae Vent.

Rocas expuestas.

Xerófila, fotófila, saxícola, basófila.

Toda la Península Ibérica.

Circumpolar y atlántica.

Orthotrichum rupestre Schw.

subsp. sturmii (Hopp. et Hornsch.) Boul.

O. sturmii Hopp. et Hornsch.

Rocas calizas expuestas.

Xerófila, fotófila, saxícola, indiferente.

Toda la Península Ibérica.

Circumboreal disyunta.

Orthotrichum Iyelii Hook et Tayl.

Sobre Crataegus (ALlORGE, V. et P., 1946).

Sobre ramas y troncos de Crataegus. Muy frecuente.

Xerófila, mesófila, esciófila, corticícola, indiferente.

Toda la Península Ibérica.

Circumboreal.

\section{EUBRYALES}

BRYACEAE

Bryum bicolor Lindb.

Fisuras de rocas con suelo.

Xerófila, fotófila, termófila, basófila.

En toda la Península Ibérica.

Submediterránea-subatlántica. 


\section{Bryum canariense Brid.}

Cornisas terrosas umbrías de rocas calizas. A $1.000 \mathrm{~m}$.

(ALLORGE, V. et P., 1946).

Xero-mesófila, esciófila, terrícola, basófila.

Diversos puntos de la Península.

Submediterránea-subatlántica.

\section{Bryum capillare L. ex Hedw.}

En rocas calizas con suelo.

Mesófila, esciófila, terri-saxícola, indiferente.

En toda la Península Ibérica.

Circumboreal disyunta.

\section{Bryum capillare Hedw.}

subsp. torquescens (De Not) Kindb.

B. torquescens Bruch.

En suelos calizos. A $1.200 \mathrm{~m}$.

Mesófila, esciófila, terri-saxícola, humícola, indiferente o basófila.

En toda la Península Ibérica.

Submediterránea-subatlántica.

Bryum capillare Hedw.

fo. longipilum Podp.

B. capillare Hedw.

var. longipilum Moenk.

Torcal de Antequera (ALLORGE, V. et P., 1946).

Roca caliza con suelo.

Mesófila, esciófila, terri-saxícola, indiferente.

Diversos puntos de la Península Ibérica.

Submediterránea-subatlántica.

Pohlia drummondii (C. Mull.) Andrews in Grout.

Sumergida en aguas frías en el nacimiento de la Villa.

Mesófila, esciófila, terrícola, indiferente.

Diversas localidades de la Península Ibérica.

Circumpolar. 


\section{LEUCODONTACEAE}

\section{Antitrichia californica Sull.}

En rocas calizas umbrías y sobre Crataegus (ALLORGE, V. et P., 1946). Rocas calizas y ramas de Crataegus.

Mesófila, esciófila, saxi-corticícola, indiferente.

Centro y Sur.

Mediterránea con disyunción americano-pacífica.

Antitrichia curtipendola (Hedw.) Brid.

Torcal de Antequera (ALLORGE, V. et P., 1946).

Rocas calizas umbrías. A $1.200 \mathrm{~m}$.

Mesófila, esciófila, saxícola, acidófila.

Parte septentrional de la Península Ibérica.

Subatlántica en Europa.

Leucodon sciuroides (Hedw.) Schw.

var. morensis (Schw.) De Not.

Torcal de Antequera (ALLORGE, V. et P., 1946).

En rocas calizas y troncos de Crataegus.

Xerófila, foto-esciófila, corticícola, indiferente.

En toda la Península Ibérica.

Mediterránea.

Pterogonium gracile (Hedw.) Sm.

P. ornithopodioides (Huds.) Lindb.

Torcal de Antequera (ALLORGE, V. et P., 1946).

Sobre rocas calizas y corteza de Crataegus.

Mesófila, foto-esciófila, saxícola, acidófila.

En toda la Península Ibérica.

Submediterránea disyunta. 


\section{NECKERACEAE}

Leptodon smithii (Hedw.) Web. et Mohr.

Sobre rocas y corteza de Crataegus.

Mesófila, esciófila, saxi-corticícola, calcícola.

En toda la Península Ibérica.

Mediterráneo-atlántica disyunta.

Neckera crispa Hedw.

Torcal de Antequera (ALLORGE, V. et P., 1946).

No ha sido encontrada, probablemente confundida con Neckeradelphus menziesii. Mesófila, esciófila, saxícola, calcícola.

En toda la Península Ibérica.

Mediterránea montana o subatlántica.

Neckeradelphus menziesii (Hook.) Steere

Neckera turgida Jur.

Rocas calizas umbrías y protegidas.

Mesófila, esciófila, saxícola, calcícola.

En toda la Península Ibérica.

Mediterránea montana o subatlántica.

Thamnobryum alopecurum (Hedw.) Nieuwl.

Thamnium alopecurum (Hedw.) B. e.

En fisuras de rocas rezumantes. A $1.100 \mathrm{~m}$.

Higrófila, esciófila, saxícola, preferentemente calcícola.

Distintos puntos de la Península Ibérica.

Mediterránea-atlántica en Europa.

\section{HYPNOBRYALES}

\section{AMBLYSTEGIACEAE}

Hygroamblystegium tenax (Hedw.) Jenn.

Amblystegium tenax (Hedw.) C. Jens.

En aguas corrientes en el Nacimiento de la Villa.

Higrófila, foto-esciófila, saxícola, indiferente.

Diversos puntos de la Península Ibérica.

Circumpolar. 
Leptodictyum riparium (Hedw.) Warnst.

Sumergido en aguas corrientes y frías. Nacimiento de la Villa.

Higrófila, foto-esciófila, terri-saxícola, indiferente.

Diversos puntos de la Península Ibérica.

Circumboreal disyunta.

Platyhypnidium riparioides (Hedw.) Dix.

$P$. rusciformis (Neck.) Fleish.

En aguas corrientes en el Nacimiento de la Villa.

Reófila, foto-esciófila, saxícola, indiferente.

En toda la Península Ibérica.

Circumboreal, casi cosmopolita.

\section{BRACHYTECIACEAE}

Brachytecium velutinum (Hedw.) B. e.

En rocas calizas no expuestas y ramas de Crataegus.

Mesófila, esciófila, terri-saxi-corticícola, indiferente.

Común en la Península Ibérica.

Circumboreal.

Camptothecium aureum (Lag.) $\mathrm{Br}$.

Torcal de Antequera (ALLORGE, V. et P., 1946).

En rocas calizas expuestas.

Xerófila, fotófila, terri-arenícola, basófila.

En toda la Península Ibérica.

Circumboreal.

Cirriphyllum crassinervium (Tayl.) Loeske \& Fleish.

En grietas de rocas calizas umbrías.

Mesófila, esciófila, saxícola, indiferente.

Norte, Cataluña, Baleares, Cádiz, Granada.

Subatlántica.

Homalothecium sericeum (Hedw.) B. e.

Torcal de Antequera (ALLORGE, V. et P., 1946).

En rocas calizas.

Meso-xerófila, fotófila, saxi-corticícola, basófila.

Común en toda la Península Ibérica.

Atlántico-mediterránea. 
Oxyrrhynchium praelongum (Hedw.) Warnst.

Rocas rezumantes umbrías.

Higro-mesófila, esciófila, terri-saxícola, indiferente.

Cataluña, Granada, León, Logroño, Vascongadas y Portugal.

Subatlántica disyunta.

Oxyrrhynchium pumilum (Wils.) Loesk.

En sue'os calizos. A $1.100 \mathrm{~m}$.

Mesófila, esciófila, terri-saxícola, indiferente.

Barcelona, Baleares, Cádiz, Gerona, Vascongadas y Portugal.

Subatlántica.

Oxyrrhynchium swartzii (Turn.) Warnst.

En rocas calizas.

Mesófila, esciófila, terrícola, indiferente.

Diversos puntos de la Península Ibérica.

Circumboreal.

Rhynchostegiella tenella (Dicks.) Limpr.

Rocas rezumantes. A $1.200 \mathrm{~m}$.

Mesófila, esciófila, saxícola, basófila.

Diversos puntos de la Península.

Submediterránea.

Plasteurhynchium meridionale (B. e.) Fleish. in Broth.

Torcal de Antequera (ALLORGE, V. et P., 1946).

En fisuras de rocas.

Xero-mesófila, foto-esciófila, saxícola, calcícola.

Región mediterránea, País Vasco y Asturias.

Mediterráneo-atlántica.

Plasteurhynchium striatulum (Spr.) Fleish. in Broth.

Torcal de Antequera (ALLORGE, V. et P., 1946).

En rocas calizas umbrías.

Mesófila, esciófila, saxícola, calcícola.

Diversos puntos de la Península Ibérica.

Mediterráneo-subatlántica. 


\section{ENTODONTACEAE}

Pseudoscleropodium purum (Hedw.) Fleish.

En suelos temporalmente inundados, en el Nacimiento de la Villa.

Mesófila, esciófila, terri-humícola, indiferente.

En toda la Península Ibérica.

Circumboreal.

HYPNACEAE

Ctenidium molluscum (Schimp.) Mitt.

Torcal de Antequera (ALLORGE, V. et P., 1946).

En rocas calizas poco expuestas.

Mesófila, foto-esciófila, terri-saxícola, calcícola.

Toda la Península Ibérica.

Circumboreal.

HEPATICAE

MARCHANTIALES

TARGIONIACEAE

Targionia hypophylla L.

Común en taludes margosos y suelos calizos.

Xerófila, fotófila, terri-saxícola, basófila.

En toda la Península Ibérica.

Cosmopolita.

GRIMALDIACEAE

Reboulia hemisphaerica (L.) Raddi.

Torcal de Antequera (ALLORGE, V. et P., 1946).

Xerófila, fotófila, terrícola, basófila.

En toda la Península Ibérica.

Cosmopolita. 


\section{MARCHANTIACEAE}

\section{Lunularia cruciata (L.) Dum}

\section{L. vulgaris $\mathrm{L}$.}

Torcal de Antequera (ALLORGE, V. et P., 1946).

En suelos calizos muy húmedos.

Higro-mesófila, esciófila, terrícola, indiferente.

En toda la Península Ibérica.

Circumboreal.

Riccia sorocarpa Bisch.

En suelos húmedos. A $1.200 \mathrm{~m}$.

Meso-xerófila, fotófila, terrícola, indiferente.

En toda la Península Ibérica.

Circumboreal.

\section{JUGERMANIALES \\ PELLIACEAE}

Pellia fabbroniana Raddi.

$P$. calycina Nees.

Borde de arroyo en el Nacimiento de la Villa.

Hidrófila, foto-esciófila, terri-humícola, basófila preferentemente.

Toda la Península.

Circumboreal.

\section{RADULACEAE}

\section{Radula lindbergiana Got.}

Sobre rocas rezumantes. A $1.200 \mathrm{~m}$.

Mesófila, esciófila, saxícola, acidófila.

La Coruña, Pontevedra, Lugo y Algarve.

Euroasiática, en las latitudes mediterráneas.

\section{MADOTHECACEAE}

Madotheca platyphylla (L.) Lindb.

Torcal de Antequera (ALLORGE, V. et P., 1946).

Xero-mesófila, foto-esciófila, corticícola, saxícola, indiferente.

Toda la Península Ibérica.

Circumboreal mesoterma. 


\section{FRULLANIACEAE}

\section{Frullania dilatata Dum.}

Sobre ramas de Crataegus.

Xero-mesófila, foto-esciófila, corticíco’a, indiferente.

Toda la Península Ibérica.

Euro-asiática.

\section{LEJEUNEACEAE}

\section{Cololejeunea rossettiana (Mass.) Schiff.}

En rocas rezumantes, A $1.200 \mathrm{~m}$.

Mesófila, esciófila, saxícola, muscícola, calcícola.

Extremadura y algunos puntos de la Península Ibérica.

Submediterránea-subatlántica.

\section{ESTUDIO BRIOSOCIOLÓGICO}

Solo se han considerado aquellas comunidades donde intervienen briófitos y realmente hay una separación tajante entre comunidades briofíticas y liquénicas. En cuanto a comunidades de suelo al estar éste muy colonizado por plantas superiores, la vegetación briofítica se refugia en fisuras de rocas, donde la vegetación superior es seleccionada, no obstante, no hemos creído oportuno efectuar un estudio sociológico de estos nichos ecológicos por considerarlos poco representativos.

El presente estudio se refiere a las siguientes comunidades:

\section{VEGETACIÓN DE ROCAS EXPUESTAS}

Las condiciones climatológicas son tan extremas, sobre todo lo referente a factores como insolación, temperatura... que la vegetación muscinal solo se presenta en superficies de orientación N. o bien poco soleadas. En ambientes soleados y verdaderamente expuestos, la roca queda solo colonizada por liquenes.

Los inventarios tomados (cuadro núm. 1) revelan la existencia de la asociación Orthotricho-Grimmietum pulvinatae Stodiek, 1937 (Schistidion apocarpi, Schistidietalia apocarpi, Tortulo-Homalothecietea sericei), clase esta última, dada por Hertel como provisional en 1974. 
ORTHOTRICHO-GRIMMIETUM PULVINATAE Stodiek 1937

$\begin{array}{llrrrrrr}\text { Número de inventario } \ldots \ldots \ldots \ldots \ldots \ldots \ldots & 1 & 2 & 3 & 4 & 5 & 6 \\ \text { Superficie }\left(\mathrm{cm}^{2}\right) & \ldots \ldots \ldots \ldots \ldots \ldots \ldots \ldots \ldots \ldots \ldots & 400 & 600 & 800 & 800 & 800 & 600 \\ \text { Cobertura }(\%) & \ldots \ldots \ldots \ldots \ldots \ldots \ldots \ldots \ldots \ldots & 50 & 30 & 30 & 20 & 30 & 30 \\ \text { Inclinación }\left({ }^{\circ}\right) & \ldots \ldots \ldots \ldots \ldots \ldots \ldots \ldots \ldots \ldots \ldots \ldots & 60 & 45 & 90 & 90 & 90 & 90\end{array}$

Características de asociación:

$\begin{array}{llcccccc}\text { Grimmia pulvinata ......................... } & 2 & 2 & 2 & 1 & 2 & - \\ \text { Orthotrichum anomalum var. saxatile } & \ldots & + & + & 1 & + & - & 2\end{array}$

Características de unidades superiores

(Schistidion apocarpi, Schistidietalia apocarpi, Tortulo-Homalothecietea sericei.)

$\begin{array}{lcccccc}\text { Schistidium apocarpum subsp. atrofuscum } & 3 & 1 & - & 2 & 1 & - \\ \text { Tortula muralis .............................. } & + & + & 1 & + & + & +\end{array}$

Homalothecium sericeum ................... - $+-t_{1}$

Compañeras

Orthotrichum cupulatum var. sardagnum - $1-1-2$

Orthotrichum cupulatum .................. - $-2--$

Orthotrichum rupestre ssp. sturmii ..... - $-2-{ }_{-}-$

Crossidium squamigerum ................. - $--t_{+}+$

Barbula acuta .............................. - -

Aloina aloides .............................. - $-{ }_{-}-+$

Barbula fallax ........................... - - $-{ }_{-}+$

\section{VEGETACIÓN DE ROCAS PROTEGIDAS}

En la base de rocas verticales, hasta una altura que en las situaciones más favorecidas no supera un metro, la vegetación pulvinular de Orthotricho-Grimmietum pulvinatae, es sustituida por una comunidad donde el dominio es de grandes pleurocárpicos como Neckeradelphus menziesii, Leucodon morensis, Homalothecium sericeum, entre otros. Se trata pues de una comunidad más mesófila que la anterior, donde si bien las temperaturas son relativamente más altas durante el verano, la insolación es prácticamente nula, manteniéndose un cierto grado de humedad. 
Para estos ambientes, proponemos la asociación Neckeradelphetum menziesii caracterizada por Leucodon morensis, Antitrichia californica, especies de extensa área y Neckeradelphus menziesii que aunque sus índices de abundancia-dominancia no son muy altos, su carácter mediterráneomontano la hace buena característica diferencial dentro de la alianza Neckerion complanatae de distribución centro europea. En el cuadro núm. 2 se exponen los inventarios tipos que caracterizan a la asociación.

Damos como inventario tipo (syntypus) el núm. 3.

CUADRO N. ${ }^{\circ} 2$

NECKERADELPHETUM MENZIESII nova.

$\begin{array}{llllllllll}\text { Número de inventario } & . . . \ldots \ldots \ldots \ldots \ldots \ldots & 1 & 2 & 3 & 4 & 5 & 6 & 7\end{array}$

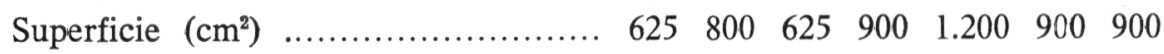

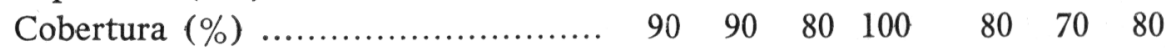

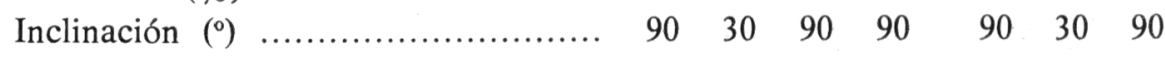

Características de asociación

Neckeradelphus menziesii ............. $14 \begin{array}{lllll} & 1 & 2 & 1 & 2 \\ +\end{array}$

$\begin{array}{lllllllll}\text { Antitrichia californica .................. } & 1 & 2 & 2 & 3 & 3 & 2 & 2\end{array}$

Leucodon sciuroides var. morensis ...

Características de unidades superiores (Neckerion complanatae, Neckeretalia complanatae, Tortulo-Homalothecietea sericei.)

Homalothecium sericeum ............. $1+121-$

Madotheca platyphylla ................ 2 - $22+-$

Compañeras

Leptodon smithii ........................ $-+\quad-\quad-\quad \begin{array}{llll}1 & 1 & 1\end{array}$

Brachythecium velutinum ............. - $-\infty-++$

Oxyrrhynchium swartzii ............... - $--_{-}+-$

Antitrichia curtipendola ................ - $1---$

Pterogonium gracile ................... - -+--

Grimmia pulvinata ................... - + - - - +

Orthotrichum anomalum var. saxatile $--_{-}-{ }_{-}+$ 


\section{VEGETACIÓN DE ROCAS REZUMANTES}

Una rápida visita a la sierra del Torcal de Antequera hace casi imposible pensar en la presencia de rocas rezumantes, pero la propia configuración cárstica favorece la presencia de grandes embudos por donde el agua se filtra permaneciendo sus paredes rezumantes y con un alto grado de humedad ambiental. El factor mínimo a superar por las especies que se instalan en tales sustratos es la luz, en algunos casos hemos tomado inventarios a profundidad de aproximadamente cuatro metros. Los inventarios del cuadro núm. 3 ponen de manifiesto la gran selección que el ambiente esciófilo efectúa sobre las especies que componen esta comunidad, generalmente muy abundantes en briófitos.

Hemos considerado como características de la asociación CololejeuneoRhynchostegietum tenellae, que proponemos: Cololejeunea rossettiana y Rhynchostegiella tenella y como características de unidades superiores, Fissidens minutulus y Eucladium verticillatum. Hertel incluye estas comunidades esciófilas en la alianza Fissidention pusillae que él da como provisional, sin inc'uirla en ninguna unidad superior. Nosotros y dada la presencia de Eucladium verticillatum a la que hemos considerado como característica de unidad superior, creemos que esta alianza estaría relacionada en nuestras latitudes con la clase Adiantetea, agrupando en la alianza Fissidentión pusillae estas comunidades muy esciófilas.

Damos como inventario tipo (syntypus) el núm. 1.

CUADRO N. 3

COLOLEJEUNEO-RHYNCHOSTEGIETUM TENELLAE nova

\begin{tabular}{|c|c|c|c|c|}
\hline Número de inventario & 1 & 2 & 3 & 4 \\
\hline uperficie $\left(\mathrm{cm}^{2}\right)$ & 2.500 & 900 & 600 & 600 \\
\hline bertura $(\%)$ & 90 & 80 & 90 & 80 \\
\hline nclinación $\left({ }^{\circ}\right)$ & 90 & 90 & 80 & 90 \\
\hline
\end{tabular}

Características de asociación

Cololejeunea rossettiana ................. $1+{ }_{+}+{ }_{1}$

Rhynchostegiella tenella ................. $1+\begin{array}{llll} & \\ 2 & 1\end{array}$

Características de unidades superiores (Fissidention pusillae)

Eucladium verticillatum ................ $3 \quad 2 \quad-\quad 2 \quad-$

Fissidens minutulus .................... $24 \quad 2 \quad 1 \quad-$ 
Compañeras

Oxyrrhinchium praelongum

Fissidens cristatus .........................

Radula lindbergiana ......................

Thamnobryum alopecurus ..............
Oxyrrhinchium pumilum ................ + -

\section{VEGETACIÓN EPIFITICA}

La vegetación arbustiva representada en su mayor parte por Crataegus monogyna Jacq. se encuentra sumamente colonizada por comunidades liquénicas y briofíticas que presentan una distribución muy distinta, las zonas basales y medias de los arbustos son colonizadas por briófitos, mientras que las ramas más externas lo son por comunidades liquénicas. La asociación Orthotricheto-Antitrichietum californicae de la que exponemos en el cuadro núm. 4 los inventarios, presenta una composición florística que nos recuerda mucho a la que se vio en rocas protegidas, diferenciándose fundamentalmente en la presencia de Orthotrichum lyelii y Frullania dilatata así como en los índices de abundancia-dominancia de algunas de las especies características.

Orthotrichum lyelii y Antitrichia californica, características de asociación, colonizan la parte media-basal de los arbustos, hacia abajo el Orthotrichum lyelii es desalojado por los pleurocárpicos que se hacen dominantes, mientras que hacia arriba Orthotrichum lyelii y Frullania dilatata son los que se ponen en contacto con la población liquénica.

Tras la revisión del trabajo Etude phytosociologique de groupements de bryophytes de la Brenne, Indre-France, efectuado por LECOINTE; la posición sintaxonómica de esta comunidad dada por ALLORGE en 1935, creemos que estaría dentro de la alianza Frullanion dilatatae en la subalianza Fabronienion pusillae, vicariante mediterránea de Tortulenion laevipilae, a pesar de no estar representada en el Torcal la Fabronia pusilla Raddi, sí lo están otras especies características de esta subalianza como Leptodon smithii y Pterogonium gracile. 
ORTHOTRICHETO-ANTITRICHETO CALIFORNICAE Allorge 1935

$\begin{array}{llrrrrr}\text { Número de inventario } \ldots \ldots \ldots \ldots \ldots \ldots . & 1 & 2 & 3 & 4 & 5 \\ \text { Cobertura }(\%) & \ldots \ldots \ldots \ldots \ldots \ldots \ldots \ldots \ldots \ldots . & 90 & 90 & 80 & 80 & 80 \\ \text { Superficie }\left(\mathrm{cm}^{2}\right) & \ldots \ldots \ldots \ldots \ldots \ldots \ldots \ldots \ldots & 1.500 & 1.500 & 600 & 600 & 600 \\ \text { Inclinación }\left({ }^{\circ}\right) \ldots \ldots \ldots \ldots \ldots \ldots \ldots \ldots \ldots \ldots & 90 & 90 & 80 & 70 & 90\end{array}$

Características de asociación

Orthotrichum lyelii .................... $+t \quad \begin{array}{llll}1 & - & 2\end{array}+$

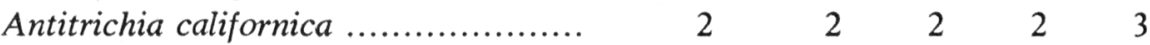

Características de unidades superiores (Fabronienion pusillae, Frullanion dilatatae, Leucodontetalia, Hypnetea uncinatii)

Leptodon smithii

$\begin{array}{ccccc}1 & 2 & 2 & 2 & 3 \\ 2 & + & 2 & 1 & 1\end{array}$

Leucodon sciuroides var. morensis ...

Frullania dilatata

Pterogonium gracile

Compañeras

Orthotrichum anomalum var. saxatile

Brachythecium velutinum

$+\quad+\quad-\quad-\quad-$

Barbula rigidula

\section{ESQUEMA SINTAXONÓMICO}

Cl. Tortulo-Homalothecietea sericei cl. prov. Hertel 1974

Ord. Schistidietalia apocarpi (Jezek \& Vondracek 1962) Hertel 1974

Al. Schistidion apocarpi Jezek \& Vondracek 1962

As. Orthotricho-Grimmietum pulvinatae Stodiek 1937

Ord. Neckeretalia complanatae Jezek \& Vondracek 1962

Al. Neckerión complanatae Hadac \& Smarda 1944

As. Neckeradelphetum menziesii nova

Cl. ?

Ord. ?

Al. Fissidention pusillae Hertel 1966

As. Cololejeuneo-Rhynchostegietum tenellae nova

Cl.? Hypnetea uncinatii Lecointe 1975

Ord. Leucodontetalia Von Hubschmann 1952

Al. Frullanion dilatatae Lecointe 1975

Subal. Fabronienion pusillae Barkman 1958

As. Orthotricheto-Antitricheto californicae Allorge 1935 


\section{BIBLIOGRAFIA}

Allorge, V. et P. 1946. Notes sur la flore bryologique de la Peninsule Iberique X. Muscinées du Sud et de l'Est de l'Espagne. Rev. Bryol. et Lichenal. París.

Allorge, V. et P. 1947. Essai de Bryogeographie de la Peninsule Iberique. Enciclopedie Biogeographique et Ecologique. París.

Augier, J. 1966. Flore de Bryophytes. Ed. P. Lechevalier. París.

Barkamann, J. 1969. Phytosociology and ecology of cryptogamic epiphytes. Van Gorcum and Comp. N. V. Assen. Holanda.

Bonos, A. 1968. Bryogeographie und Bryoflora Ungarns. Akademiai Kiadó. Budapest.

Cabanas, R. 1960. El Torcal de Antequera un típico Karst de mesa. Est. Geogr., n. ${ }^{\circ}$ 78, págs. 63-82. Madrid.

Casares-Gil, A. 1919. Flora ibérica, Briofitas I, Hepáticas. Trab. Mus. Nac. C. Nat. Madrid.

Casares-Gil, A. 1932. Flora ibérica, Briofitas II, Musgos. Trab. Mus. Nac. C. Nat. Madrid.

Delgado, J. 1975. Estudio Hidrogeológico del Torcal de Antequera (Málaga). Departamento de Hidrogeología. Facultad de Ciencias de Granada (Tesina).

Demaret, F. et Castagne, E. 1959. Flore general de Belgique. Bryophites, vol II-III. Bruselas.

Dixon, H. N. 1970. The Student's handbook of British mosses. 2. ${ }^{a}$ Ed. Londres.

DunK, KLAUS VON der. 1972. Moosqesellschaften im Bereich des Sandsteinkeupers in Mittel-und Oberfranken. Bericht, Band XIV, Bayreuth.

García Chicano, J. L. y Delgado, M. 1971. Génesis y tipos de suelos que se desarrollan sobre las rocas calizas del Torcal de Antequera (Málaga). Anales de Edafología y Agrobiología. Tomo XXX, números 1-2. Madrid.

Hertel, E. 1974. Epilithische Moose und Moosqesellschaften im nordöstlichen Ballern. Naturwissenschfteinche Gesellschaft Bayreuth. Tomo I. Bayreuth.

Hilde, N. y Navratil, S. Mooskleinqesellschaften der Städte. Nova Hedwigia. Band II, fac. 3, págs. 425-463. Weinhein.

Hubschmann, A. 1971. Bryosoziologische Studien auf Der insel Madeira. Nova Hedwigia. Band XXII. Heft 1-2. Weinheim.

Hubschmann, A. 1967. Uber die Moosgesellschaften und das Vorkommen der Moose in den übriqen Pflanzenqesellschaften des Moseltales. Sonderdruck aus Schriftenreihe für Vegetationskunde. Vol. 2, págs. 63-121. Bas Godesberg.

Husnot, T. 1922. Muscologia Gallica. 2. ${ }^{a}$ Ed. Asser. Co. Amsterdam.

Karlsruhe, G. P. 1965. Die Moosqeselschaften der Wutachschlucht. Mitt band Landesver Naturkunde u Naturschutz. Neve Folge VIII (4).

Lecointe, A. 1975. Etude Phytosociologique des groupemenst de bryophytes epiphites de la Brenne (Indre-France). Documents phytosociologique. Fas 9-14. Lille. Francia.

Monkemeyer, W. 1927. Die Laubmoose Europas. Kryptogamen Flora Rabenhorst's. Leipzig.

MulleR, K. 1957. Die Lebermoose. Kryptogamen Flora Rabenhorst's. Leipzig.

Nyнolm, E. 1975. Moss Flora of Fennoscandia. The Botanical Society of Lund. Estocolmo.

Poppera, J. 1973. Bryum generis monographiae. Prodromus I-II. Czechoslovak Academy of Sciences. Praga.

PhilipPI, G. 1972. Die Moosvegetation der Wälder in der Rheinaue Zwischen Basel und Mannhein. Beitr. naturk. Forsch. SüdwDtl. Band 31, págs. 5-64. Karlsruhe.

Wilmanns, O. 1962. Rindenbewohnende Epiphytenqemeinschaften in Südwestdentschland. Beith. naturk. Forsch SW-Deutschl. Bd. XXI, Vol. 2, págs. 87-164. Karlsruhe. 


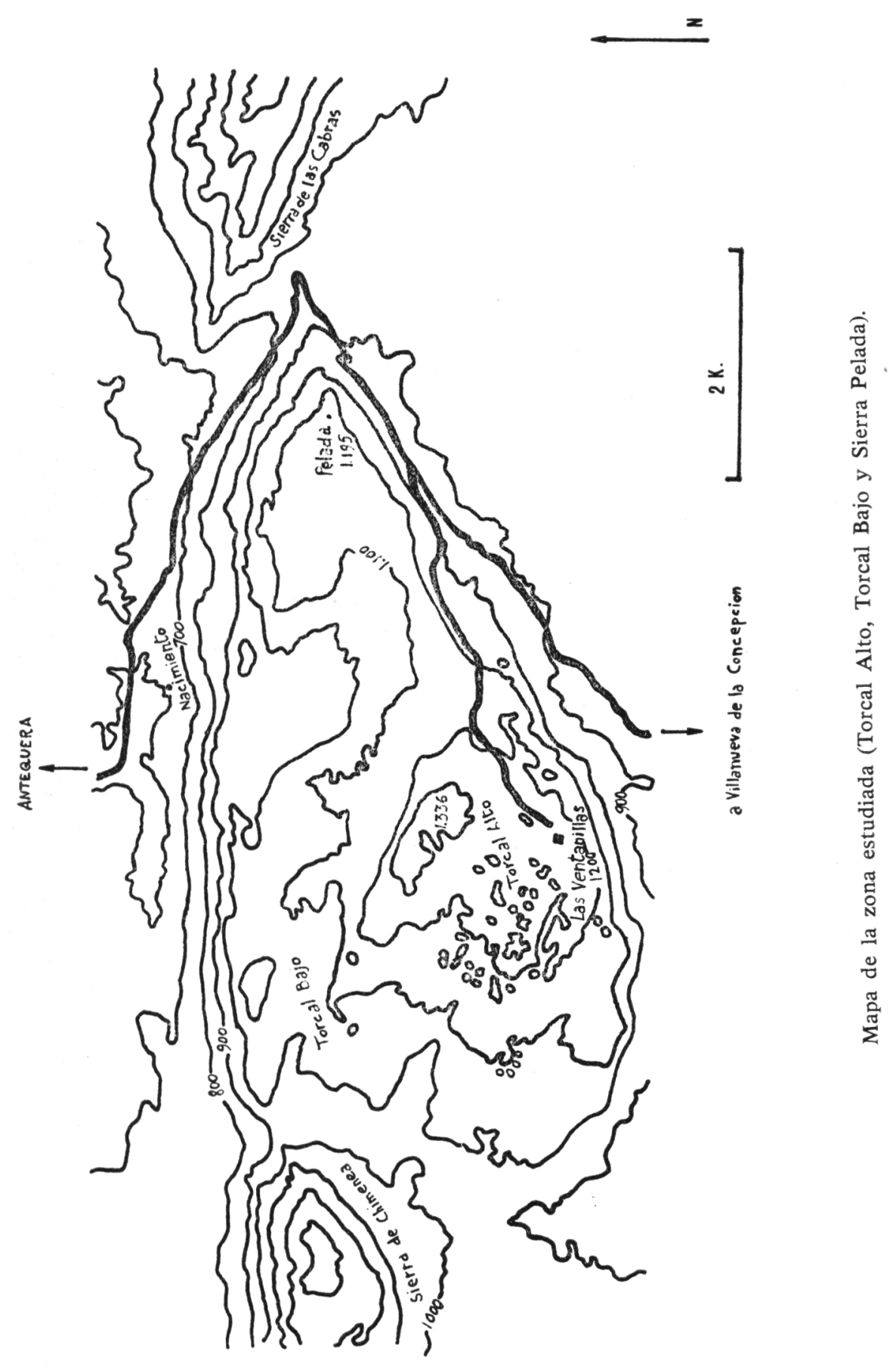




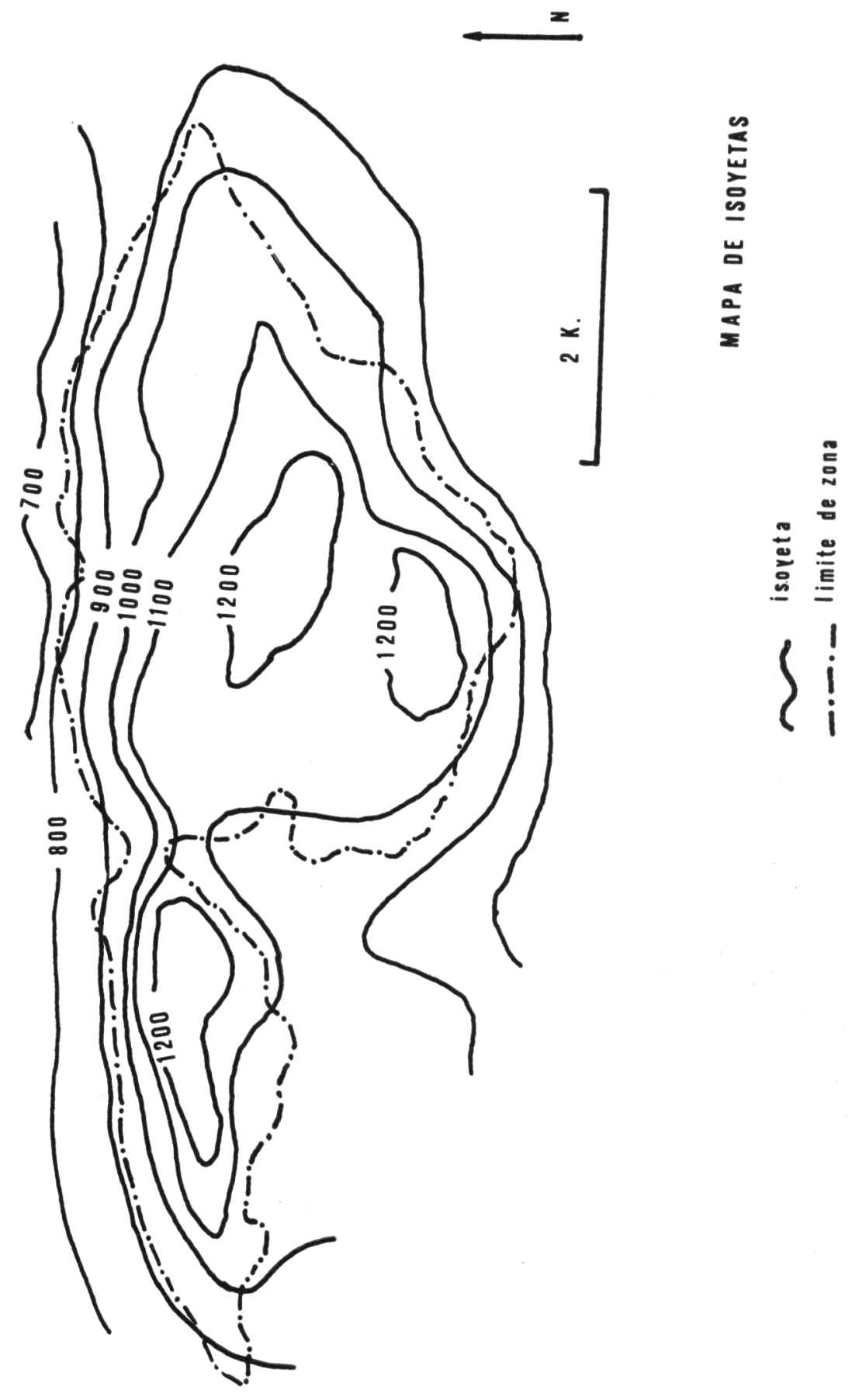




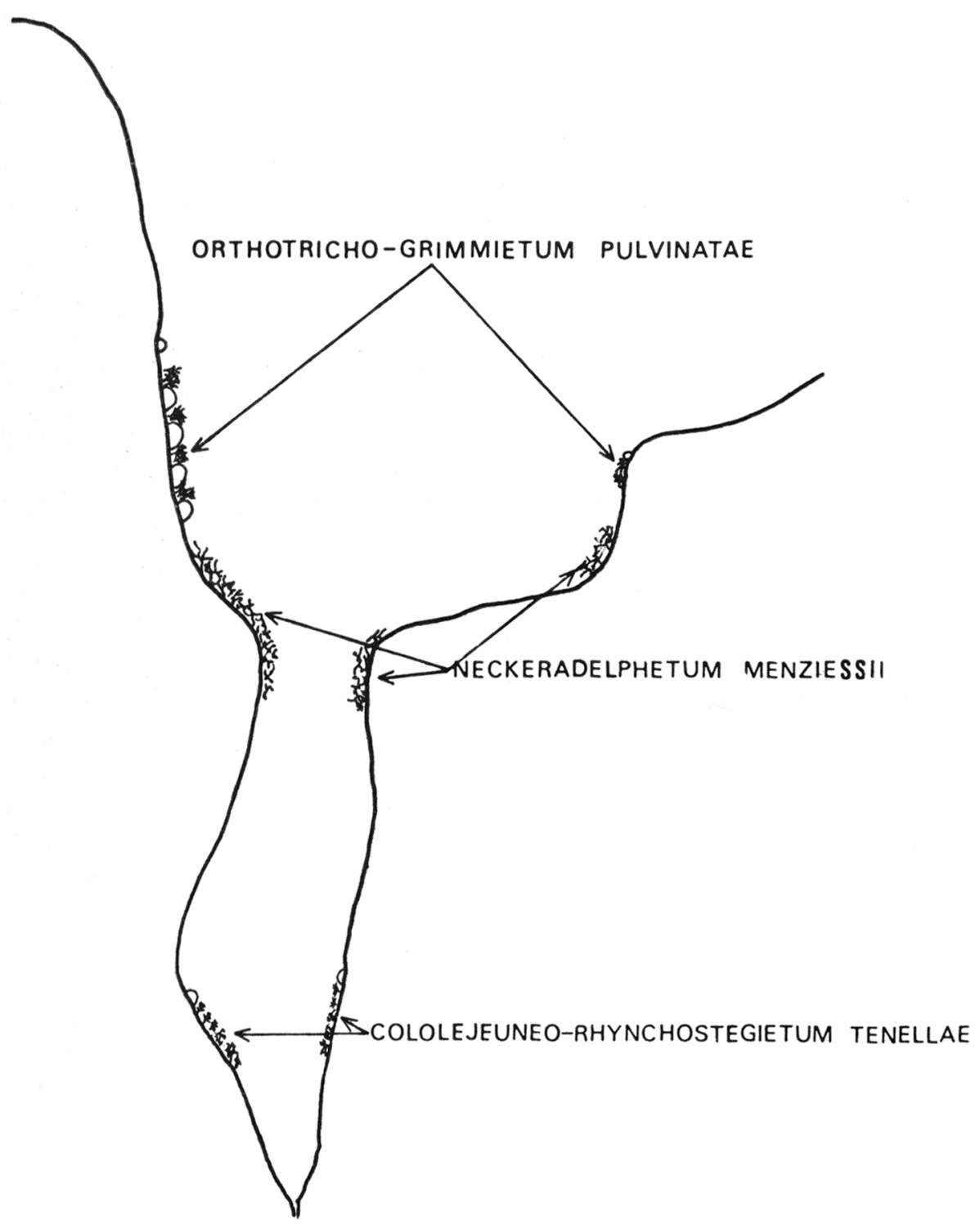

Esquema de las comunidades de briófitos que se asientan sobre rocas. 


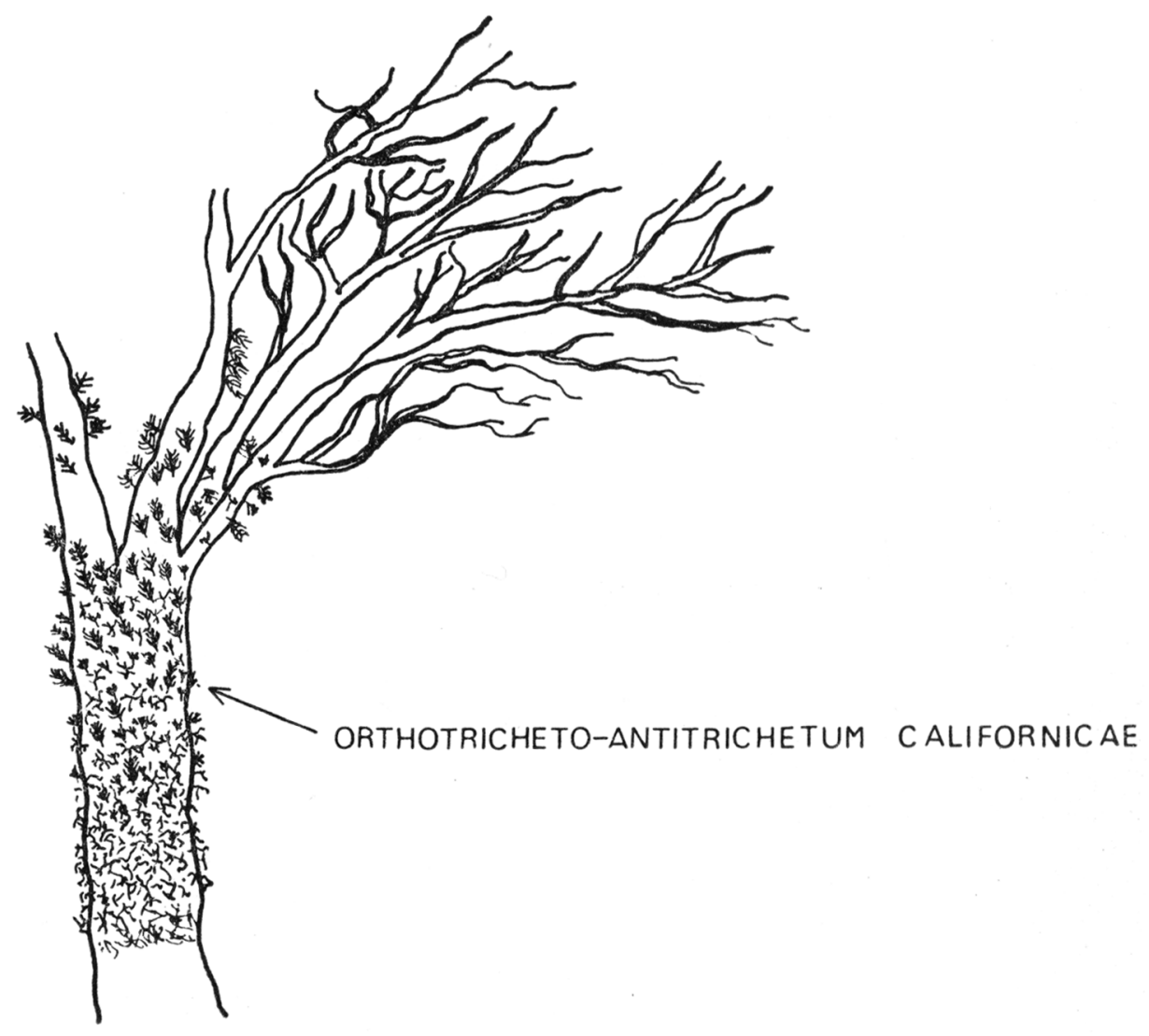

Esquema de la comunidad epifítica de briófitos que se encuentra sobre Crataegus monogyna Jacq. 